\title{
Release characteristics of bone-like hydroxyapatite/poly amino acid loaded with rifapentine microspheres in vivo
}

\author{
YUWU LIU, JIMING ZHU and DIANMING JIANG \\ Department of Orthopedic Surgery, The First Affiliated Hospital of Chongqing Medical University, \\ Chongqing 400016, P.R. China
}

Received May 28, 2016; Accepted April 5, 2017

DOI: $10.3892 / \mathrm{mmr} .2017 .6747$

\begin{abstract}
Bone-like hydroxyapatite/poly amino acid (BHA/PAA) is a potential bone repair material. Rifapentine-loaded poly(lactic-co-glycolic acid) microspheres (RPMs) are bioactive and efficient controlled-release delivery systems used in vitro. The aim of the present study was to investigate the in vivo drug release characteristics of RPM-loaded BHA/PAA on a rabbit model of bone defect. RPM was combined with BHA/PAA to obtain the drug-loaded, slow-releasing bioactive material. Bone defects were generated in New Zealand white rabbits and the rabbits were then implanted with RPM-loaded BHA/PAA. High-performance liquid chromatography (HPLC) was used to determine the concentrations of rifapentine in the plasma and the local muscle tissues of the treated rabbits. Hematoxylin and eosin (H\&E) staining and biochemical analyses were performed to elucidate potential side effects of RPM-loaded BHA/PAA on the heart, liver and kidney histopathology and functions of the treated rabbits. The biocompatibility and osteogenic ability of RPM-loaded BHA/PAA was evaluated by H\&E staining. The results demonstrated that the material was completely degraded and absorbed at 12 weeks following implantation and new trabecular bone and cartilage tissues had formed. The in vivo release tests revealed that RPM-loaded BHA/PAA exhibited sustained release profiles of rifapentine and the drug concentration in the muscle tissues remained higher than the minimum inhibitory concentration of rifapentine against Mycobacterium tuberculosis for as long as 12 weeks. In addition, RPM-loaded BHA/PAA had no long-term side effects to the heart, liver and kidney of the treated rabbits. In conclusion, the present study demonstrated that RPM-loaded BHA/PAA
\end{abstract}

Correspondence to: Professor Dianming Jiang, Department of Orthopedic Surgery, The First Affiliated Hospital of Chongqing Medical University, 1 Youyi Road, Chongqing 400016, P.R. China E-mail:dmj571026@126.com

Key words: rifapentine-loaded poly(lactic-co-glycolic acid) microsphere, bone-like hydroxyapatite/poly amino acid, osteoarticular tuberculosis, bone defect, drug release, side effect slowly and continuously released rifapentine in vivo and exhibited no side effects on heart, liver and kidney tissues and function. Furthermore, RPM-loaded BHA/PAA promoted new bone formation, while it was gradually degraded and absorbed. The present study provided a theoretical basis for the potential advancement in developing novel treatments for osteoarticular tuberculosis.

\section{Introduction}

Tuberculosis is a chronic, transmittable disease caused by Mycobacterium tuberculosis. Approximately one-third of the world's population is estimated to be infected with tuberculosis (1-3). Osteoarticular tuberculosis is recognized as the most common site of extrapulmonary tuberculosis, accounting for $\sim 35-50 \%$ of extrapulmonary tuberculosis and 3-5\% of the total tuberculosis $(4,5)$. The traditional oral anti-tuberculosis agents do not result in satisfactory treatment of osteoarticular tuberculosis, due to the low drug concentration achieved locally in the tuberculosis lesion and a series of side effects $(6,7)$.

The combination of drug-delivery systems and bone tissue repair appears to be the most promising option for advancement in osteoarticular tuberculosis treatment (8). At present, the reconstruction implants used for the treatment of bone defects include autogenous bone, allograft bone and artificial bone. However, autogenous bone and allograft bone have clinical limitations in the treatment of osteoarticular tuberculosis (9). For example, they exhibit low drug loading capacity and have limited tendency to release the drugs in a sustained manner. To overcome these disadvantages, researchers developed artificial bone materials, which have become commonly used carriers for anti-tuberculosis drugs in the treatment of osteoarticular tuberculosis (9).

Poly(lactic-co-glycolic acid) (PLGA) is widely used as a carrier material for controlled drug release (10). It exhibits good biocompatibility and biodegradability, and delays the release of drugs. Rifapentine is a highly effective, first-line anti-tuberculosis drug. It is synthesized in one step from rifampin. Rifapentine has an antibacterial spectrum similar to that of rifampin; however, it has a stronger antibacterial activity to $M$. tuberculosis, a milder adverse reaction and its drug elimination half-life is longer than rifampin (11-14). Rifapentine is able to effectively penetrate into infected bone, dead bone, inflammatory cells and biofilms formed by bacteria 
to eradicate bacterial infections (15). Furthermore, rifapentine prevents drug resistance (15). An in vitro study from Wu et al (16) reported that rifapentine-loaded PLGA microspheres (RPMs) are bioactive and efficient controlled-release delivery systems, with great promise towards the treatment of osteoarticular tuberculosis (16).

Hydroxyapatite (HA) is a widely-used replacement material for bone tissue repairing $(17,18)$. Bone-like hydroxyapatite (BHA) is a type of carbonate HA, that is similar to the composition and structure of normal bone. BHA exhibits good biocompatibility, safety and osteogenic activity (19-21). Compared with HA, BHA has stronger biological activity and faster degradability (19). However, pure BHA is not conducive to processing. When BHA is combined with poly amino acid (PAA), which has a similar molecular structure to collagen, its strength and mechanical properties are enhanced. A previous study from our group has demonstrated that BHA/PAA is effective in osteogenesis and reconstruction of long segmental bone defects both in vivo and in vitro (22). Recently, Yan et al (23) reported that RPM-loaded BHA/PAA is effective in the treatment of rabbit chronic osteomyelitis induced by Staphylococcus aureus. However, the effects of RPM-loaded BHA/PAA on osteoarticular tuberculosis have not been examined to date.

In the present study, the in vivo drug release characteristics of RPM-loaded BHA/PAA were evaluated on rabbit model of bone defects. Furthermore, the osteogenesis induction ability and the side effects of RPM-loaded BHA/PAA were investigated in vivo. The present study may provide a novel approach for the clinical treatment of osteoarticular tuberculosis.

\section{Materials and methods}

Preparation of materials. RPMs were prepared through an oil-in-water emulsion solvent evaporation method, as previously described $(16,23)$. Briefly, $50 \mathrm{mg}$ of rifapentine (Jinan Mingxin Pharmaceutical Co., Ltd., Sichuan, China) was dissolved into the polymer solution and $200 \mathrm{mg}$ of PLGA (Jinan Daigang Biomaterial Co., Ltd., Jinan, China) was dissolved in $10 \mathrm{ml}$ of dichloromethane. RPMs were prepared with an entrapment efficiency of $85.78 \pm 2.00 \%$, an actual drug loading of $17.16 \pm 0.40 \%$ and a mean diameter of $25.267 \mu \mathrm{m}$. The BHA/PAA materials (National Nanomaterial Company of Sichuan University, Chengdu, China) were prepared using the standard atmospheric pressure solution method (24). RPMs were composited with BHA/PAA to make RPM-loaded BHA/PAA, with a size of $15 \times 5 \times 5 \mathrm{~mm}^{3}$, a weight of $750.50 \pm 8.54 \mathrm{mg}$ and an aperture size of $100-500 \mu \mathrm{m}$.

Rabbit model with bone defects. The animal experiments were approved by the Animal Care Committee of Chongqing Medical University (Chongqing, China). A total of 66 male New Zealand white rabbits at the age of 4 months and weighing 2.5-3 kg were obtained from the Experimental Animal Center of Chongqing Medical University. All rabbits were housed at $17-21^{\circ} \mathrm{C}$, with a $12-\mathrm{h}$ light/dark cycle and $30-70 \%$ relative humidity. They had free access to water and food. The rabbits were anesthetized by intravenous injection of pentobarbital $(30 \mathrm{mg} / \mathrm{kg})$ in the ear margin. The bilateral femoral condyle was shaved and disinfected. An $\sim 5 \mathrm{~mm}$-depth hole was made by a $4 \mathrm{~mm}$ drilling bit from the femoral lateral condyle to the interior condyle. Rabbits in the BHA/PAA group $(n=6)$ or RPM-loaded BHA/PAA group $(n=48)$ were implanted with BHA/PAA or RPM-loaded BHA/PAA materials in the bilateral bone holes, while rabbits in the blank group $(n=6)$ were not treated with any materials (Fig. 1). Rabbits in the normal group $(n=6)$ did not receive any treatment. Following washing with saline, the wounds were closed with sutures layer by layer.

Hematoxylin and eosin $(H \& E)$ staining. A total of 6 rabbits from each group was sacrificed at the indicated times. Then, the bone, heart, liver and kidney tissues were collected and fixed in $10 \%$ paraformaldehyde overnight, followed by embedding in paraffin. The paraffin-embedded tissues were cut into $5 \mu \mathrm{m}$ thick sections and immersed in xylene followed by a graded series of ethanol to remove the paraffin. The sections were then stained with H\&E (Beyotime Institute of Biotechnology, Shanghai, China) and observed under a light microscope (Nikon Corporation, Tokyo, Japan). Three sections were prepared and viewed for each tissue sample.

High-performance liquid chromatography (HPLC). Concentrations of rifapentine in the plasma and the local muscle tissues were determined using HPLC. All 48 rabbits in the RPM-loaded BHA/PAA group were subjected to this assay. A total of $4 \mathrm{ml}$ blood was collected from the ear margin vein and centrifuged at $10,000 \mathrm{x}$ g for $5 \mathrm{~min}$ at room temperature. The supernatant $(100 \mu \mathrm{l})$ was mixed with $1 \mathrm{ml}$ of methanol, followed by centrifugation at $10,000 \mathrm{x} \mathrm{g}$ for $10 \mathrm{~min}$ at $4^{\circ} \mathrm{C}$. Finally, $20 \mu \mathrm{l}$ of the supernatant was collected for the drug concentration assay. Muscle tissue $(\sim 1 \mathrm{~g})$ around the material implanting site was collected and homogenized, and $500 \mu \mathrm{l}$ of the homogenate was mixed with $1.5 \mathrm{ml}$ formaldehyde and vortexed for $2 \mathrm{~min}$. Following centrifugation at $10,000 \times \mathrm{g}$ for $10 \mathrm{~min}$ at $4^{\circ} \mathrm{C}, 20 \mu \mathrm{l}$ of the supernatant was collected for the drug concentration assay. The internal standard rifapentine was obtained from the National Institute for the Control of Pharmaceutical and Biological Products (Beijing, China). HPLC analysis was performed using a C18 column (250x4.6 mm; Waters Corporation, Milford, MA, USA) with the mobile phase of methanol-acetonitrile $(5: 4 \mathrm{v} / \mathrm{v})$ on a D-2000 HPLC system (Hitachi, Ltd., Tokyo, Japan). The temperature was set at $40^{\circ} \mathrm{C}$, the flow rate was $0.6 \mathrm{ml} / \mathrm{min}$ and the wavelength was $268 \mathrm{~nm}$.

Biochemical analyses. The blood samples ( $\mathrm{n}=6$ for each group) were centrifuged at $1,000 \mathrm{x} \mathrm{g}$ and $4^{\circ} \mathrm{C}$ for $10 \mathrm{~min}$, and the serum was extracted and stored at $-20^{\circ} \mathrm{C}$. The concentrations of alanine aminotransferase (ALT), aspartate aminotransferase (AST), blood urea nitrogen (BUN) and creatinine $(\mathrm{Cr})$ in the serum were measured using an Olympus AU2700 Automated Chemistry Analyzer (Olympus Corporation, Tokyo, Japan).

Statistical analysis. Statistical analysis was performed using SPSS version 19.0 statistical software (IBM Corp., Armonk, NY, USA) and the data were expressed as the mean \pm standard deviation. The significance of differences between two groups was analyzed using Student's t-test. $\mathrm{P}<0.05$ was considered to indicate a statistically significant difference. 
Table I. The weight of rabbits following surgery, kg.

\begin{tabular}{|c|c|c|c|c|c|c|}
\hline & 1 week & 2 weeks & 3 weeks & 4 weeks & 8 weeks & 12 weeks \\
\hline Normal & $2.9 \pm 0.1$ & $3.1 \pm 0.1$ & $3.3 \pm 0.2$ & $3.6 \pm 0.3$ & $4.3 \pm 0.3$ & $4.7 \pm 0.5$ \\
\hline Blank & $2.4 \pm 0.2^{\mathrm{a}}$ & $2.7 \pm 0.1$ & $3.1 \pm 0.2$ & $3.3 \pm 0.3$ & $3.8 \pm 0.3$ & $4.4 \pm 0.2$ \\
\hline BHA-PAA & $2.3 \pm 0.3^{\mathrm{a}}$ & $2.9 \pm 0.2$ & $3.1 \pm 0.1$ & $3.2 \pm 0.2$ & $3.6 \pm 0.3$ & $4.5 \pm 0.3$ \\
\hline RPM-loaded BHA/PAA & $2.3 \pm 0.2^{\mathrm{a}}$ & $2.8 \pm 0.1$ & $3 \pm 0.1$ & $3.3 \pm 0.2$ & $3.9 \pm 0.4$ & $4.2 \pm 0.3$ \\
\hline
\end{tabular}

aP $<0.05$ compared with the normal group. BHA/PAA, bone-like hydroxyapatite/poly amino acid; RPM,rifapentine-loaded poly(lactic-co-glycolic acid) microsphere.

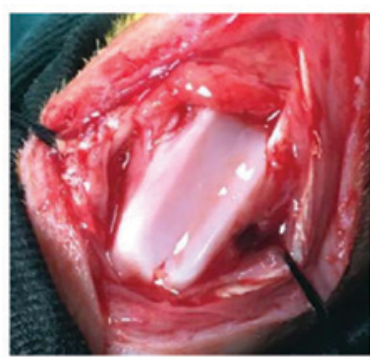

Blank

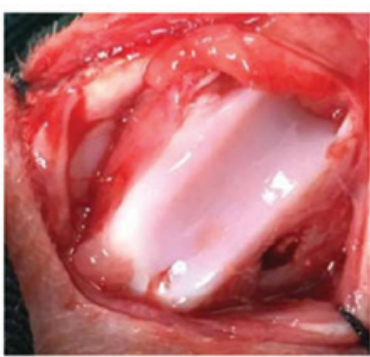

BHA-PAA

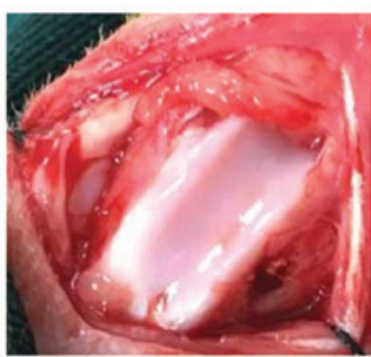

RPM-loaded BHA/PAA

Figure 1. A rabbit model of bone defects following material implantation. BHA/PAA, bone-like hydroxyapatite/poly amino acid; RPM, rifapentine-loaded poly(lactic-co-glycolic acid) microsphere.

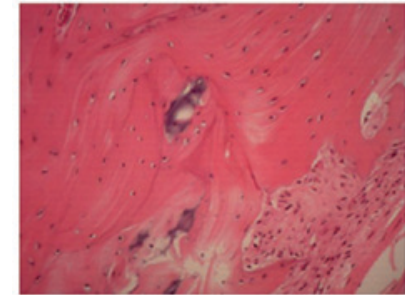

Blank

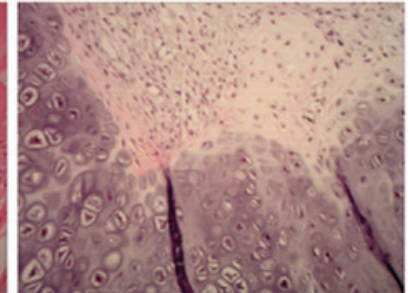

BHA/PAA

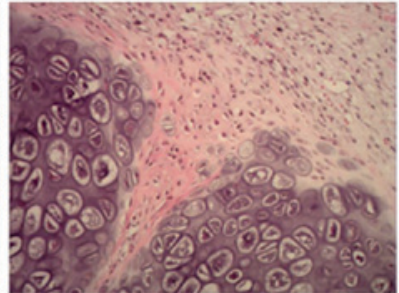

RPM-loaded BHA/PAA

Figure 2. Hematoxylin and eosin staining of bone tissue sections at 12 weeks following implantation. Magnification, x400. BHA/PAA, bone-like hydroxyapatite/poly amino acid; RPM, rifapentine-loaded poly(lactic-co-glycolic acid) microsphere.

\section{Results}

General observation after bone defect. Following surgery, all the rabbits suffered from lackluster behavior, lameness and decline in appetite; the wound was red and swollen, and the rabbits lost weight. However, no mortality was observed. One week following implantation, the rabbits gradually recovered. The weight of the rabbits in the RPM-loaded BHA/PAA group increased and returned to the normal weight after two weeks. The weights of the rabbits in the blank and BHA/PAA group also increased, but remained lower than normal (Table I). After 12 weeks, normal gait was restored in the BHA/PAA and RPM-loaded BHA/PAA groups; however, the rabbits in the blank group still suffered from lameness.

$H \& E$ staining of bone specimens. At 12 weeks following implantation, bone specimens were collected and stained with H\&E. As demonstrated in Fig. 2, a large amount of fibrous tissue was observed in the blank group. In the BHA/PAA and
RPM-loaded BHA/PAA groups, the implanted materials were degraded and absorbed, and new bone had formed.

Concentrations of rifapentine in the plasma and the local muscle tissues. The concentrations of rifapentine detected in the plasma and muscle tissues of the RPM-loaded BHA/PAA group are listed in Table II. Rifapentine concentration in the plasma samples reached the peak level at day 1 following implantation; however, it decreased significantly at day 3 and was undetectable at 2 weeks following implantation (Table II). The concentration of rifapentine in the muscle tissues around the material implanting site was also examined. The results revealed that rifapentine concentration in the muscle tissues was higher than the minimum inhibitory concentration of rifapentine against $M$. tuberculosis $(0.12-0.25 \mathrm{mg} / \mathrm{l})$ (25) for at least 12 weeks following implantation (Table II).

Side effects of RPM-loaded BHA/PAA on heart, liver and kidney. The heart, liver and kidney tissues were collected 
Table II. Concentrations of rifapentine in plasma and local muscle tissues in the RPM-loaded BHA/PAA group.

\begin{tabular}{lcccccccc}
\hline & \multicolumn{8}{c}{ Concentration of rifapentine, $\mu \mathrm{g} / \mathrm{ml}$} \\
\cline { 2 - 8 } Sample & 1 day & 3 days & 1 week & 2 weeks & 3 weeks & 4 weeks & 8 weeks & 12 weeks \\
\hline Plasma & 5.2 & 1.1 & 0.2 & 0 & 0 & 0 & 0 \\
Muscle tissues & 12.2 & 8.5 & 8 & 7.5 & 6.3 & 5.1 & 3.8 \\
\hline
\end{tabular}

BHA/PAA, bone-like hydroxyapatite/poly amino acid; RPM, rifapentine-loaded poly(lactic-co-glycolic acid) microsphere.

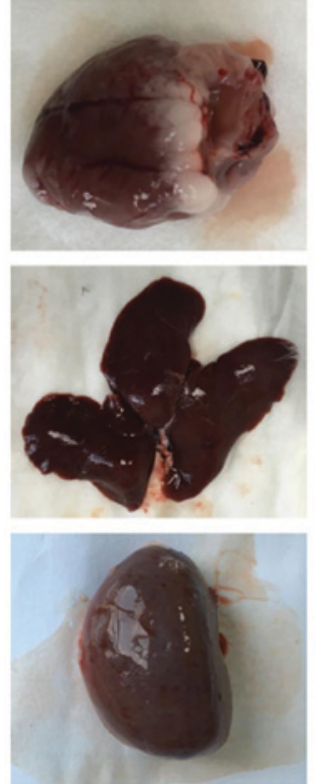

Blank
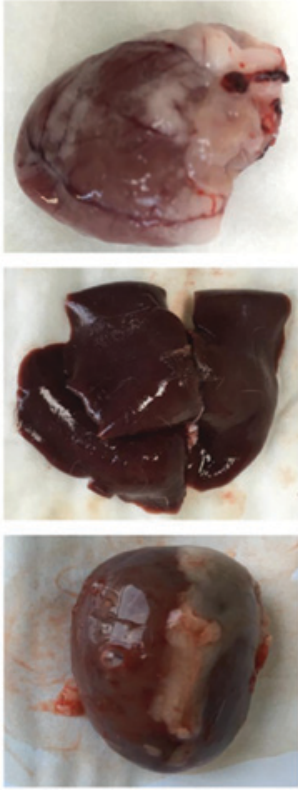

BHA/PAA

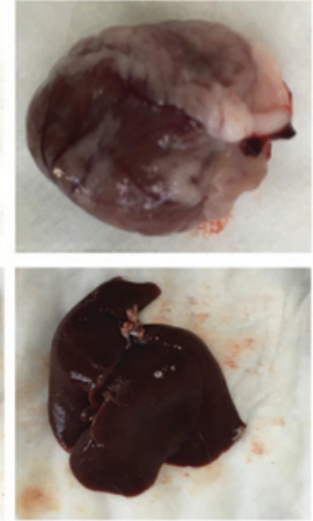

Heart

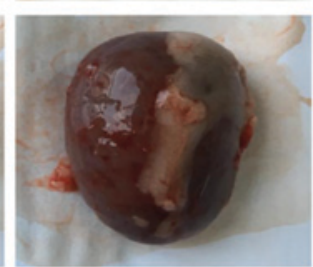

RPM-loaded BHA/PAA

Figure 3. Heart, liver and kidney specimens at 12 weeks following implantation. BHA/PAA, bone-like hydroxyapatite/poly amino acid; RPM, rifapentine-loaded poly(lactic-co-glycolic acid) microsphere.

at 12 weeks following implantation. As illustrated in Fig. 3, no significant changes were observed in the appearance of heart, liver and kidney amongst the three groups. In addition, H\&E staining revealed no histopathological abnormalities in the heart, liver and kidneys of the treated rabbits (Fig. 4). Biochemical analyses for markers of liver and kidney functions revealed that the concentrations of ALT, AST, BUN and Cr in the serum were significantly increased at day 1 and 3 following implantation; however, serum concentrations for all four markers returned to normal levels within 1 week (Table III).

\section{Discussion}

BHA/PAA is a polar polymer which has great potential as a bone repair material. It combines the biocompatibility and bone conduction of BHA with the mechanical properties of PAA, to induce osteogenesis. Previous studies have reported that the polar groups in BHA/PAA, including- $\mathrm{OH},-\mathrm{NH}_{2}$ and $-\mathrm{COOH}$, promote the colonization of bone cells on the material (26). In addition, the porous network structure and surface roughness of BHA/PAA provide a biochemical environment to promote osteoblast proliferation, differentiation, migration, adhesion and promote bone repair and regeneration (27-29). A previous study by Yan and Jiang (22) confirmed that BHA/PAA exhibits good osteogenesis activity both in vitro and in vivo; in vitro, BHA/PAA promoted the growth, adhesion and calcium nodule formation of MG63 bone osteosarcoma cells, while in an animal model of radial bone defect, the implanted BHA/PAA material could be fused with the host bone and new bone gradually formed. In the present study, the BHA/PAA material was completely degraded and absorbed at 12 weeks following implantation and new trabecular bone and cartilage had formed. The present results were consistent with previous studies from our group $(22,23)$ and confirmed that BHA/PAA is a good bone graft substitute material with good biological compatibility and capable of osteogenic induction.

RPM was first generated by our research group (16) and preliminary experiments have characterized the physiochemical properties of RPMs. RPMs were spherical with rough surfaces. The in vitro drug release studies revealed that following an initial rapid drug release, rifapentine release gradually decreased and $\sim 80 \%$ of the encapsulated rifapentine was released following $\sim 4$ weeks of incubation. Furthermore, RPMs were able to eliminate $S$. aureus in vitro. These results indicated that RPMs were bioactive and efficient controlled-release delivery systems, and may exhibit a great 
Table III. Concentrations of ALT, AST, BUN and Cr in serum.

\begin{tabular}{llccrc}
\hline & Experimental group & ALT $(\mathrm{U} / \mathrm{l})$ & AST $(\mathrm{U} / \mathrm{l})$ & BUN $(\mathrm{mmol} / \mathrm{l})$ & Cr $(\mu \mathrm{mol} / \mathrm{l})$ \\
\hline \multirow{2}{*}{1 day } & Blank & $48.82 \pm 7.05$ & $55.65 \pm 9.09$ & $7.78 \pm 1.25$ & $131.97 \pm 25.62$ \\
& BHA/PAA & $88.56 \pm 6.08^{\mathrm{b}}$ & $92.37 \pm 10.46^{\mathrm{a}}$ & $15.11 \pm 2.05^{\mathrm{b}}$ & $245.11 \pm 25.95^{\mathrm{b}}$ \\
& RPM-loaded BHA/PAA & $98.76 \pm 11.68^{\mathrm{b}}$ & $113.67 \pm 18.21^{\mathrm{b}}$ & $15.92 \pm 1.84^{\mathrm{b}}$ & $258.12 \pm 30.57^{\mathrm{b}}$ \\
3 days & BHA/PAA & $81.58 \pm 11.67^{\mathrm{a}}$ & $82.05 \pm 7.05^{\mathrm{a}}$ & $10.07 \pm 2.12$ & $218.91 \pm 30.34^{\mathrm{a}}$ \\
& RPM-loaded BHA/PAA & $85.05 \pm 9.05^{\mathrm{b}}$ & $85.34 \pm 7.02^{\mathrm{a}}$ & $11.15 \pm 2.01$ & $222.14 \pm 25.98^{\mathrm{a}}$ \\
1 week & BHA/PAA & $60.66 \pm 7.25$ & $70.02 \pm 7.11$ & $9.21 \pm 1.88$ & $200.74 \pm 28.79$ \\
& RPM-loaded BHA/PAA & $65.28 \pm 7.88$ & $73.27 \pm 8.05$ & $9.35 \pm 2.05$ & $198.12 \pm 25.67$ \\
& BHA/PAA & $54.25 \pm 6.92$ & $54.25 \pm 5.88$ & $7.15 \pm 1.68$ & $142.28 \pm 29.22$ \\
& RPM-loaded BHA/PAA & $52.43 \pm 7.02$ & $60.13 \pm 7.67$ & $8.02 \pm 1.22$ & $155.28 \pm 20.95$
\end{tabular}

${ }^{a} \mathrm{P}<0.05$ and ${ }^{\mathrm{b}} \mathrm{P}<0.01$ compared with the blank group. ALT, alanine aminotransferase; AST, aspartate aminotransferase; BUN, blood urea nitrogen; Cr, creatinine; BHA/PAA, bone-like hydroxyapatite/poly amino acid; RPM, rifapentine-loaded poly(lactic-co-glycolic acid) microsphere.
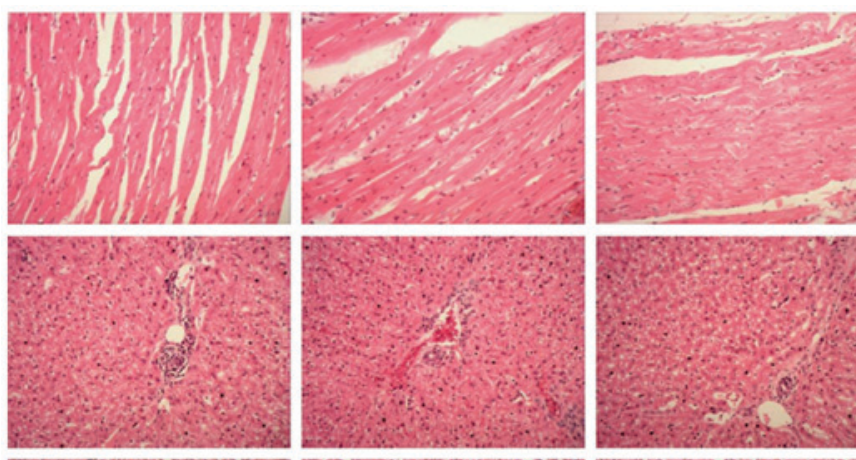

Heart
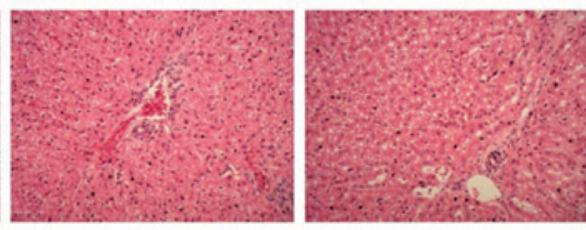

Liver

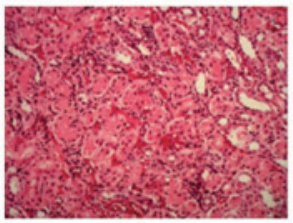

Blank

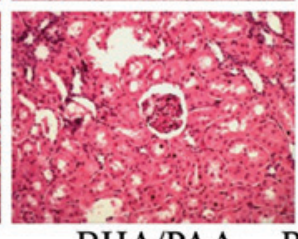

BHA/PAA

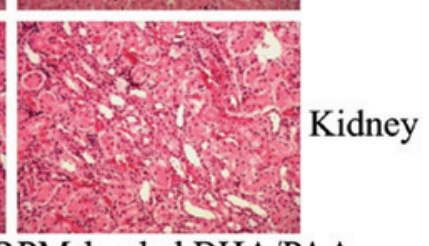

RPM-loaded BHA/PAA

Figure 4. Hematoxylin and eosin staining of heart, liver and kidney tissue sections at 12 weeks following implantation. Magnification, x400. BHA/PAA, bone-like hydroxyapatite/poly amino acid; RPM, rifapentine-loaded poly(lactic-co-glycolic acid) microsphere.

potential in the treatment of osteoarticular tuberculosis (16). In the present study, BHA/PAA was used as a carrier of RPM to prepare a novel sustained-release drug material.

A previous study has demonstrated the curative effect of RPM-loaded BHA/PAA in the treatment of rabbit chronic osteomyelitis induced by $S$. aureus (23). The in vitro experiments demonstrated that RPM-loaded BHA/PAA was able to slowly release antibiotics and inhibited bacterial growth effectively for up to 5 weeks. In vivo, RPM-loaded BHA/PAA effectively eradicated the bacterial infection, induced osteogenesis and promoted new bone formation while the material was gradually degraded and absorbed. The present study aimed to investigate the curative effect of RPM-loaded BHA/PAA in the treatment of osteoarticular tuberculosis. The in vivo release tests demonstrated that RPM-loaded BHA/PAA exhibited sustained release profiles of rifapentine and the drug concentration in the muscle tissues remained higher than the minimum inhibitory concentration of rifapentine against
M.tuberculosis for as long as 12 weeks. Furthermore, the H\&E staining and biochemical analyses indicated that RPM-loaded BHA/PAA had no long-term side effects to the heart, liver and kidneys of the treated rabbits.

In conclusion, the present study revealed for the first time that RPM-loaded BHA/PAA may be a useful material for treating osteoarticular tuberculosis. RPM-loaded BHA/PAA promoted new bone formation, while it was gradually degraded and absorbed. Furthermore, rifapentine was released in a sustained manner from this material and no side effects were observed in the heart, liver and kidney of the treated animals. The present results may therefore provide useful new tools towards improving the treatment of osteoarticular tuberculosis.

\section{Acknowledgements}

This research was supported by the National Natural Science Foundation of China (no. 81171685). 


\section{References}

1. Dye $\mathrm{C}$ and Williams BG: The population dynamics and control of tuberculosis. Science 328: 856-861, 2010.

2. Chen M, Gan H and Remold HG: A mechanism of virulence: Virulent Mycobacterium tuberculosis strain H37Rv, but not attenuated H37Ra, causes significant mitochondrial inner membrane disruption in macrophages leading to necrosis. J Immunol 176: 3707-3716, 2006.

3. Barry CE III, Boshoff HI, Dartois V, Dick T, Ehrt S, Flynn J, Schnappinger D, Wilkinson RJ and Young D: The spectrum of latent tuberculosis: Rethinking the biology and intervention strategies. Nat Rev Microbiol 7: 845-855, 2009.

4. Nagashima H, Yamane K, Nishi T, Nanjo Y and Teshima R: Recent trends in spinal infections: Retrospective analysis of patients treated during the past 50 years. Int Orthop 34: 395-399, 2010.

5. WHO global tuberculosis control report 2010. Summary. Cent Eur J Public Health 18: 237, 2010.

6. Sequeira W, Co H and Block JA: Osteoarticular tuberculosis: Current diagnosis and treatment. Am J Ther 7: 393-398, 2000.

7. Ge Z, Wang Z and Wei M: Measurement of the concentration of three antituberculosis drugs in the focus of spinal tuberculosis Eur Spine J 17: 1482-1487, 2008.

8. Saifullah B, Hussein MZ and Hussein Al Ali SH: Controlled-release approaches towards the chemotherapy of tuberculosis. Int J Nanomedicine 7: 5451-5463, 2012.

9. Moore WR, Graves SE and Bain GI: Synthetic bone graft substitutes. ANZ J Surg 71: 354-361, 2001.

10. Freitas S, Merkle HP and Gander B: Microencapsulation by solvent extraction/evaporation: Reviewing the state of the art of microsphere preparation process technology. J Control Release 102: 313-332, 2005.

11. Aristoff PA, Garcia GA, Kirchhoff PD and Showalter HD: Rifamycins-obstacles and opportunities. Tuberculosis (Edinb) 90: 94-118, 2010

12. Keung A, Eller M, McKenzie K and Weir S: Single and multiple dose pharmacokinetics of rifapentine in man: Part II. Int J Tuberc Lung Dis 3: 437-444, 1999.

13. Bemer-Melchior P, Bryskier A and Drugeon HB: Comparison of the in vitro activities of rifapentine and rifampicin against Mycobacterium tuberculosis complex. J Antimicrob Chemother 46: 571-576, 2000.

14. Assandri A, Ratti B and Cristina T: Pharmacokinetics of rifapentine, a new long lasting rifamycin, in the rat, the mouse and the rabbit. J Antibiot (Tokyo) 37: 1066-1075, 1984.

15. Chan JG, Bai X and Traini D: An update on the use of rifapentine for tuberculosis therapy. Expert Opin Drug Deliv 11: 421-431, 2014

16. Wu J,Zuo Y,Hu Y, Wang J, Li J, Qiao B and Jiang D: Development and in vitro characterization of drug delivery system of rifapentine for osteoarticular tuberculosis. Drug Des Devel Ther 9: 1359-1366, 2015

17. Gentile P, Chiono V, Boccafoschi F, Baino F, Vitale-Brovarone C, Vernè E, Barbani N and Ciardelli G: Composite films of gelatin and hydroxyapatite/bioactive glass for tissue-engineering applications. J Biomater Sci Polym Ed 21: 1207-1226, 2010.
18. Roohani-Esfahani SI, Nouri-Khorasani S, Lu Z, Appleyard R and Zreiqat $\mathrm{H}$ : The influence hydroxyapatite nanoparticle shape and size on the properties of biphasic calcium phosphate scaffolds coated with hydroxyapatite-PCL composites. Biomaterials 31 : 5498-5509, 2010.

19. $\mathrm{Wu} \mathrm{Y}$ and Bose S: Nanocrystalline hydroxyapatite: Micelle templated synthesis and characterization. Langmuir 21: 3232-3234, 2005

20. Mastrogiacomo M, Scaglione S, Martinetti R, Dolcini L, Beltrame F, Cancedda R and Quarto R: Role of scaffold internal structure on in vivo bone formation in macroporous calcium phosphate bioceramics. Biomaterials 27: 3230-3237, 2006.

21. Turco G, Marsich E, Bellomo F, Semeraro S, Donati I, Brun F, Grandolfo M, Accardo A and Paoletti S: Alginate/Hydroxyapatite biocomposite for bone ingrowth: A trabecular structure with high and isotropic connectivity. Biomacromolecules 10: 1575-1583, 2009.

22. Yan L and Jiang DM: Study of bone-like hydroxyapatite/polyamino acid composite materials for their biological properties and effects on the reconstruction of long bone defects. Drug Des Devel Ther 9: 6497-6508, 2015.

23. Yan L, Jiang DM, Cao ZD, Wu J, Wang X, Wang ZL, Li YJ and Yi YF: Treatment of Staphylococcus aureus-induced chronic osteomyelitis with bone-like hydroxyapatite/poly amino acid loaded with rifapentine microspheres. Drug Des Devel Ther 9: 3665-3676, 2015 .

24. Peng XL, Li YB, Wang XJ, Yan YG, Wei J and Zhang L: Study on the soaking behaviors of nano-hydroxyapatite/polyamide 66 biomedical composite in vitro. Functional Mater 2: 253-255, 2004

25. Rastogi N, Goh KS, Berchel M and Bryskier A: Activity of rifapentine and its metabolite 25-O-desacetylrifapentine compared with rifampicin and rifabutin against Mycobacterium tuberculosis, Mycobacterium africanum, Mycobacterium bovis and M. bovis BCG. J Antimicrob Chemother 46: 565-570, 2000.

26. Bos R, van der Mei HC and Busscher HJ: Physico-chemistry of initial microbial adhesive interactions-its mechanisms and methods for study. FEMS Microbiol Rev 23: 179-230, 1999.

27. Wei G and Ma PX: Structure and properties of Nano-hydroxyapatite/polymer composite scaffolds for bone tissue engineering. Biomaterials 25: 4749-4757, 2004

28. Owen GR, Jackson J, Chehroudi B, Burt H and Brunette DM: A PLGA membrane controlling cell behaviour for promoting tissue regeneration. Biomaterials 26: 7447-7456, 2005.

29. Huang J, Zhao D, Dangaria SJ, Luan X, Diekwisch TG, Jiang G, Saiz E, Liu G and Tomsia AP: Combinatorial design of hydrolytically degradable, bone-like biocomposites based on PHEMA and hydroxyapatite. Polymer (Guildf) 54: 909-919, 2013. 